$[0.5,3.3] \mathrm{P}=0.006$ versus baseline and a decrease of $1.1 \mathrm{mmHg}[0.2,2.1], \mathrm{P}=0.02$ versus $8.7 \mathrm{micromol} / \mathrm{min}$ nitrite.

There were also significant decreases in the time to LV end-systole (LVEST, $\mathrm{P}=0.004$, with $26 \mathrm{micromol} / \mathrm{min}$ nitrite resulting in a decrease of $11 \mathrm{~ms}[4,18] \mathrm{P}=0.002$ versus baseline) and the LV end-diastolic pressure-volume relation (EDPVR, $\mathrm{P}=0.006$ ).

No significant changes in $\mathrm{dP} / \mathrm{dtmin}(\mathrm{P}=0.2)$ or tau $(\mathrm{P}=0.3)$ were seen. The changes in diastolic function occurred without
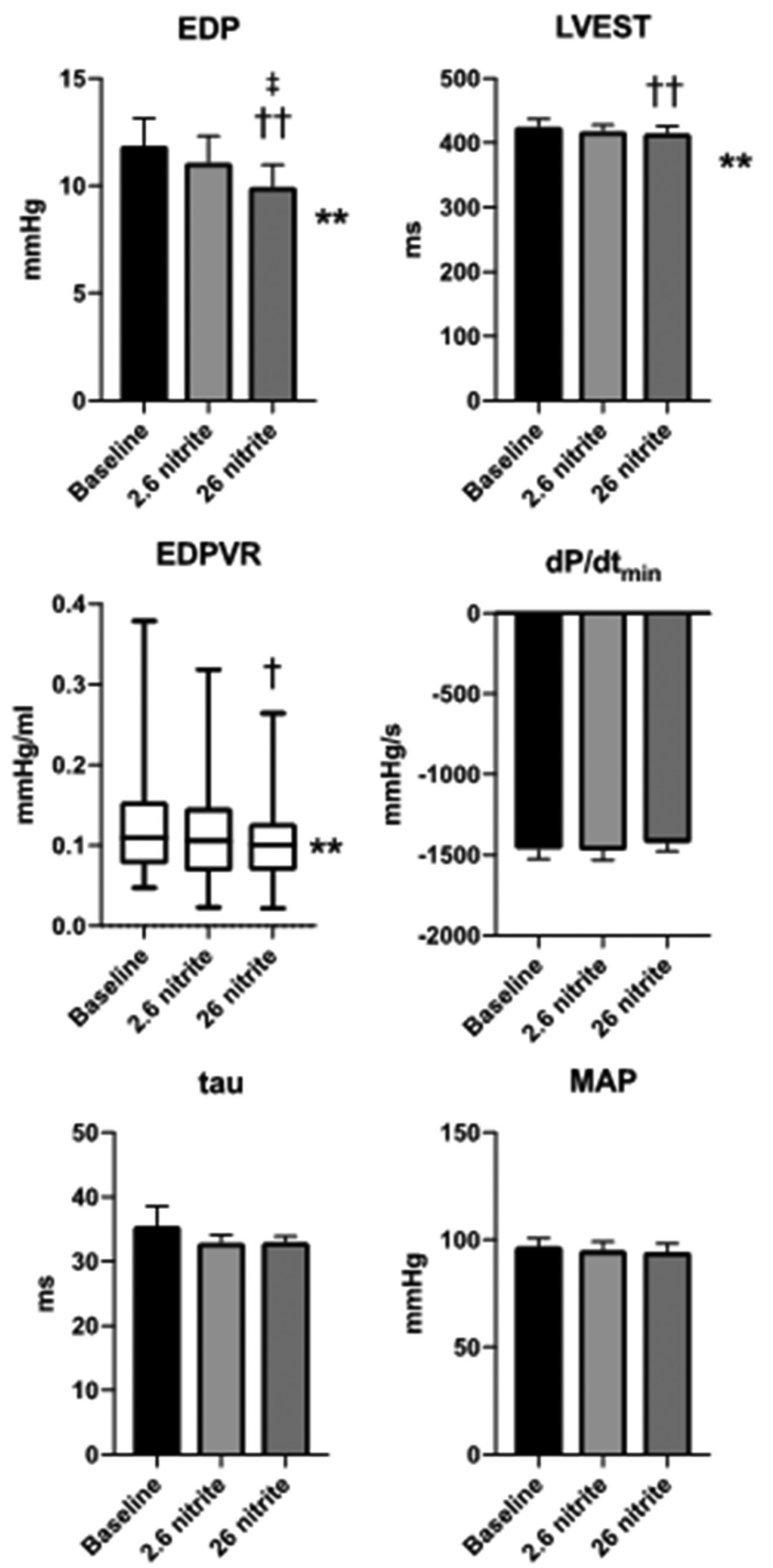

Abstract 80 Figure 1 The effect of intracoronary inorganic nitrite (8.7 and $26 \mu \mathrm{mol} / \mathrm{min}$ ) on parametersof LV diastolic function and mean arterial pressure. Bar charts expressed as mean \pm SEM

${ }^{*} \mathrm{P}<0.01$ for ANOVA. $t p<0.05$ vs baseline, $+t p<0.01$ vs baseline. $\neq p<0.05$ vs $8.7 \mu \mathrm{mol} / \mathrm{min}$ nitrite any associated change in mean arterial pressure, heart rate or $\mathrm{LV}$ systolic function (all $\mathrm{p}=\mathrm{NS}$ ).

Conclusion Intracoronary Inorganic nitrite exerts a direct effect on LV diastolic function in humans, independent of changes in systolic function or blood pressure. Inorganic nitrite affects both the onset of relaxation and LV end-diastolic properties. These data indicate that the direct cardiac effects of inorganic nitrite contribute significantly to the overall effects of systemically-delivered nitrite and have potential implications for patients with LV diastolic dysfunction and HFpEF.

Supported by the MRC, BHF and GSTT/KCL NIHR Biomedical Research Centre.

Conflict of Interest Nil

\section{WHY DO PATIENTS WITH CONCOMITANT HF AND COPD RETURN TO HOSPITAL? RISK FACTORS AND REASONS FOR SHORT-TERM READMISSION}

${ }^{1}$ Claudia Gulea*, ${ }^{2}$ Rosita Zakeri, ${ }^{3}$ Jennifer K Quint. ${ }^{1}$ National Heart and Lung Institute, Imperial College London; ${ }^{2}$ National Heart and Lung Institute, Imperial College London; ${ }^{3}$ National Heart and Lung Institute, Imperial College London

\subsection{6/heartjnl-2019-BCS.79}

Background COPD is one of the most frequent comorbidties in HF and COPD exacerbations are a competing cause of hospitalisation in this patient group. We evaluated the causes and predictors of 30-day readmission in a large population of patients hospitalised for $\mathrm{HF}$ with comorbid COPD.

Methods We identified 54,953 patients hospitalised for $\mathrm{HF}$ with a comorbid diagnosis of COPD in the National Readmissions Database in the United States, in 2012. Using Cox proportional hazards regressions we evaluated factors associated with 30-day all-cause, respiratory and cardiovascular (CV)related readmissions. The primary cause of readmission was identified using ICD-9CM codes.

Results The most common cause for readmission was CV $(50.5 \%)$, while the most frequent cause for non-CV readmission was respiratory disease (13\%). Factors associated with 30-day all-cause readmission included: Renal failure (HR 1.24, 95\% CI 1.19 - 1.30), anaemia (HR 1.14, 95\% CI 1.09 - 1.19), weight loss (HR 1.14, 95\% CI 1.05 1.24), diabetes (HR 1.09, 95\% CI 1.04 - 1.14), and index hospitalisation length of stay over 10 days (HR 1.45, 95\% CI 1.36 - 1.55, Figure 1). Respiratory-related readmission was positively associated with recent weight loss (HR 1.37, 95\% CI 1.09 - 1.71), depression (HR 1.21 , 95\% CI 1.02 1.44), obesity (HR 1.17, 95\% CI 1.02 - 1.36) and length of stay over 10 days (HR 1.86, 95\% CI 1.54 - 2.25). Major risk factors for $\mathrm{CV}$ readmission were renal failure (HR 1.42, 95\% CI 1.33 - 1.51), coronary artery disease (HR 1.18, 95\%CI 1.11 - 1.26) and diabetes (HR 1.18, 95\% CI 1.10 - 1.24).

Conclusion In patients hospitalised for $\mathrm{HF}$ with comorbid COPD, most readmissions are still due to a CV cause; however, several non-cardiac risk factors significantly contribute to readmission risk. These data highlight an opportunity to better assess and treat non-cardiac comorbidities in order to reduce readmissions in this high-risk population. 


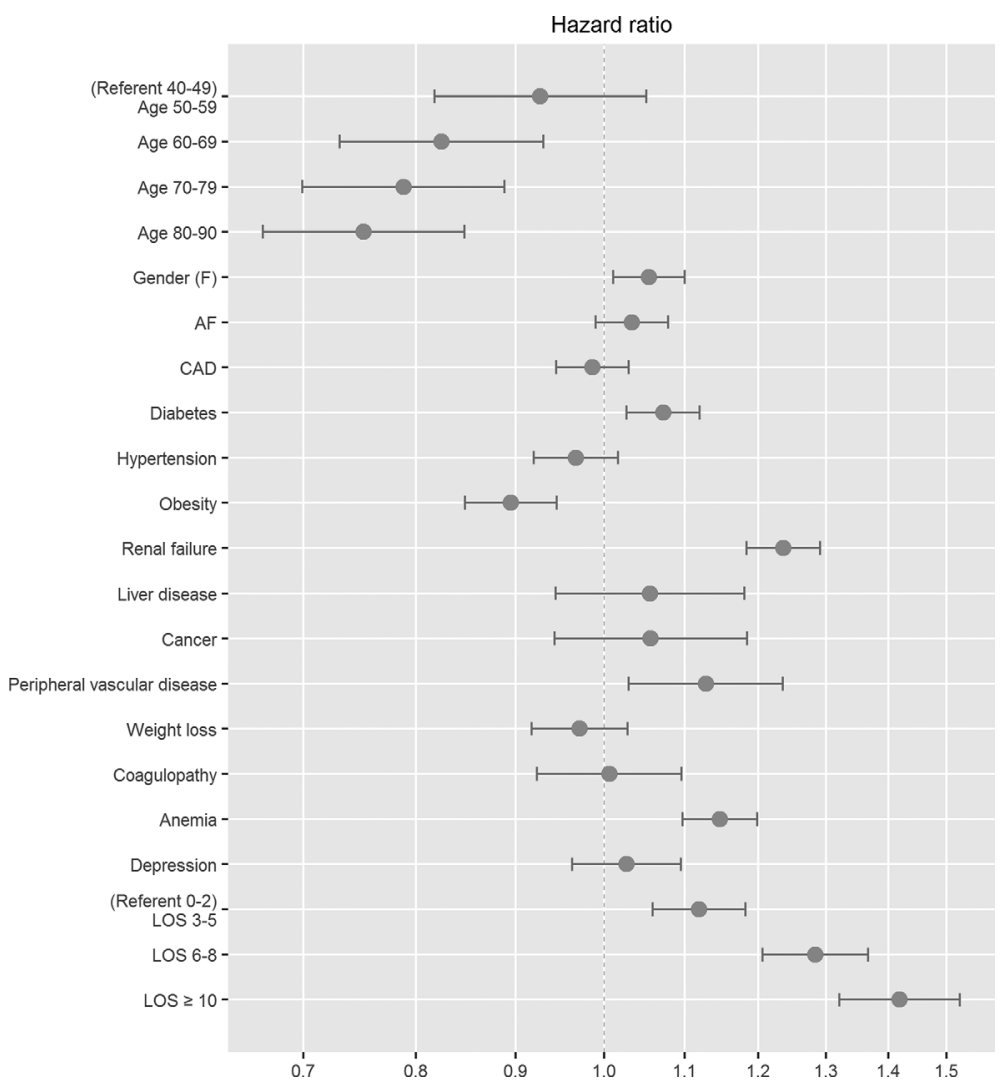

Abstract 81 Figure 1 Hazard ratio

\section{Conflict of Interest None}

\section{PEOPLE PRESCRIBED LOOP DIURETICS HAVE A POOR OUTCOME EVEN WITHOUT A DIAGNOSTIC LABEL OF HEART FAILURE}

${ }^{1}$ Jocelyn Friday*, ${ }^{1}$ Pierpaolo Pellicori, ${ }^{1}$ Richard Papworth, ${ }^{2}$ Maria Wolters, ${ }^{3}$ David McAllister, ${ }^{1}$ Sharon Kean, 'John GF Cleland. 'Robertson Centre for Biostatistics; ${ }^{2}$ University of Edinburgh; ${ }^{3}$ University of Glasgow

\subsection{6/heartjnl-2019-BCS.80}

Background Lack of a robust, practical definition of heart failure not only creates diagnostic uncertainty but limits the utility of epidemiological estimates of incidence, prevalence and prognosis on which health-care planning (both diagnosis and management) depends. Moreover, because of these uncertainties, heart failure research often focuses on patients with whom there is a high degree of certainty about the diagnosis, which may be a small fraction of the total disease burden. One important feature of heart failure is congestion and class of pharmaceutical agents is used almost exclusively (at least in the UK) for the treatment of congestion, namely loop diuretics.

Purpose We sought to describe the incidence and three-year mortality of patients with a diagnosis of heart failure and/or prescribed loop diuretic therapy in a substantial regional population.
Methods The NHS Greater Glasgow \& Clyde Health Board provides healthcare for approximately one million people. We obtained and linked records for primary and secondary health-care, prescriptions and deaths between 2010 and 2016.

We identified a heart failure diagnosis by using a list of 127 diagnostic codes from hospital (International Classification of Disease, tenth revision [ICD-10]) and primary care (Read) coding schemes. We defined incident heart failure as the first record of heart failure in primary or secondary care in any diagnostic position. We defined patients as being initiated on a loop diuretic if they received repeat prescriptions or died within 90 days of the first prescription. Patients were excluded if they died on study inclusion date; $\mathrm{n}=635$ patients.

Results Patient characteristics and three-year survival are shown in the table below. The three-year mortality for patients prescribed a loop diuretic only was $27 \%$; it was $37 \%$ for those who received a diagnosis of heart failure but were not prescribed a loop diuretic, and it was $48 \%$ for patients diagnosed with heart failure and who were also prescribed a loop diuretic (table 1 ).

Conclusion Many more patients receive a prescription for a loop diuretic than were diagnosed with heart failure.

The prognosis of patients prescribed a loop diuretic is generally poor and even worse if they also have a diagnosis of heart failure.

Conflict of Interest None 